\title{
RADIAL PULSATION ANALYSES OF DA DWARFS*
}

\author{
S. Starrfield ${ }^{\dagger}$, A. N. Cox, and S. W. Hodson \\ Theoretical Division \\ Los Alamos Sclentific Laboratory \\ Los Alamos, NM
}

\section{INTRODUCTION}

There are now twelve $\mathrm{ZZ}$ Cet 1 stars known and their propertles are summarized by Nather (1978) and McGraw (1977, 1979). They have normal DA spectra and their colors range from a B-V of 0.18 mag to 0.22 mag. This places them on the HR diagram in a region which is an extension to the white dwarfs of the Cepheld instabllity strip. This would suggest that the cause of their pulsation could be analogous to the $K$ and $\gamma$ mechanisms of the Cepheids except for the fact that the periods of the $\mathrm{ZZ}$ Cet 1 stars range from 200 to 1200 seconds, three orders of magnitude larger than the radial pulsation periods for white dwarfs.

Previous analyses of white dwarfs have shown no instabilities in the radial modes (c.f. Dzlembowsk1 1977). While Dzlembowski (1977) did find some evidence for an Instability in nonradial modes, his periods were much too short for the $2 Z$ Cet 1 variables and the particular modes that have been Identifled with some $\mathrm{ZZ}$ Ceti stars (the low order g-modes) were stable.

Therefore, we felt that it was necessary to study realistic white dwarf envelopes as carefully as possible with the new Los Alamos opacities and try to Identify any destabilizing mechanisms present in the models with a future application to a non-radial study. Our initial results whth the Los Alamos extension of Castor's (1971) 11near nonadiabatic computer program (hereafter, LNA) showed that the high order radial overtones of DA dwarfs were unstable (Cox, Hodson, and Starrfield 1979). We have now extended that work to various chemical compositions and slightly more realistic radil and report on that work in this paper.

\section{CONSTRUCTION OF THE MODELS}

The observations of the $\mathrm{ZZ}$ Cet varlables imply that their effective temperatures range from $9 \times 10^{3} \mathrm{~K}$ to $1.5 \times 10^{4} \mathrm{~K}$ (McGraw 1979). This Implies luminosities of $\sim 10^{-3} \mathrm{~L}_{\Theta}$. We chose a mass of $0.6 \mathrm{M}_{\Theta}$ and obtained radif from both the Hamada and Salpeter (1961) mass-radius relation for carbon white dwarfs with thin hydrogen envelopes and a white

*Supported In part by NSF Grant AST77-23190 to Arizona State University.

talso at Department of Physics, Arizona State University, Tempe, Az. 
dwarf cooling curve computed w1th the Kutter and Sparks (1972, see also Starrfleld, et. al. 1978) hydrodynamic stellar evolution code. The evolutionary results showed that the outer radius shrank by nearly ten percent as the dwarf cooled from $1.8 \times 10^{4} \mathrm{~K}$ to $8 \times 10^{3} \mathrm{~K}$. The different radil that we used changed the perlods and unstable modes only minimally.

One of our primary concerns was the effect of composition on the unstable modes and their perlods. Therefore, we varled the helium content in the surface layers from $Y=0.00$ to $Y=0.88$. At temperatures of $10^{6} \mathrm{~K}$ we switch to more hellum rich mixtures and then to hellum plus carbon mixtures. At densitles greater than $10^{4} \mathrm{gm} \mathrm{cm}^{-3}\left(\mathrm{~T} \sim 10^{7} \mathrm{~K}\right.$ ) we assume a pure carbon mixture. We use the Los Alamos Opacity Library (Huebner, et. al. 1977) for many mixtures at temperatures greater than $1.2 \times 10^{4} \mathrm{~K}$ ( $1 \mathrm{ev}$ ); below that value we use the Stellingwerf (1975) fit to the King tables (Cox and Tabor 1976). All models had regions of convective Instability and we used a standard mixing-length formulation (c.f. Cox and Giuli 1968) with $\ell / H_{p}=1.0$. Comparison of our model envelopes with those of Fontaine and $\operatorname{Van}$ Horn (1976) showed good agreement. We treated the luminosity variation with depth by a relationship of the form: $L(r)=(1-q) L_{s}$ where $q=M_{r} / M$ and $L_{s}$ is the surface Iuminosity of the star (Van Horn 1978, private communication). All of the envelopes were integrated to a $q$ of 0.01 .

Figure 1 shows the run of opacity, density, and temperature as a function of surface mass fraction, $1-q$, for a typical model $(10,000 \mathrm{~K})$ in which we found an instability to radial pulsations. The small irregularities in the opacity curve are the regions of composition discontinuity where we changed tables. These discontinulties have no effect on the instabilities that we have found since they lie considerably deeper into the star than the region of any pulsation driving or damping.

An Important feature of our envelopes is that there exists a significant convective region near the surface of all models with $T_{e}$ less than $10^{4} \mathrm{~K}$. In Figure 2 we show the temperature at the bottom of the convective zone as a function of the effective temperature of the star. $Y$ is the mass fraction of helium and the points for $Y=0.0$ were obtained from Fontaine and Van Horn (1976). Our $Y=0.0$ envelopes give essentially the same results. Regions at $T \sim 40,000 \mathrm{~K}$ and $150,000 \mathrm{~K}$ labeled "driving" are obtained from the LNA analysis of these envelopes. Since a strongly convective region cannot be a driving region (there is no radiation to modulate the $K$ and $\alpha$ mechanisms), it will act to stabilize those models where it extends to depths considerably below the driving region. In fact, the red edge for our envelopes does occur at prectsely those effective temperatures $\left(9-10 \times 10^{3} \mathrm{~K}\right)$ where convection carries all the luminosity. This 18 an interesting result since it has been frequently proposed that very dominant convection was the cause of the red edge of the Cepheids. We have now demonstrated that it definitely causes the red edge in the $\mathrm{ZZ}$ Cet 1 variables.

\section{RESULTS}

Our LNA analyses of the radial modes of $0.6 \mathrm{M} 0$ white dwarf envelopes with hydrogen rich surface layers show that there is an instability strip which lies at effective temperatures between $\sim 9 \times 10^{3} \mathrm{~K}$ and $1.4 \times 10^{3} \mathrm{~K}$ 

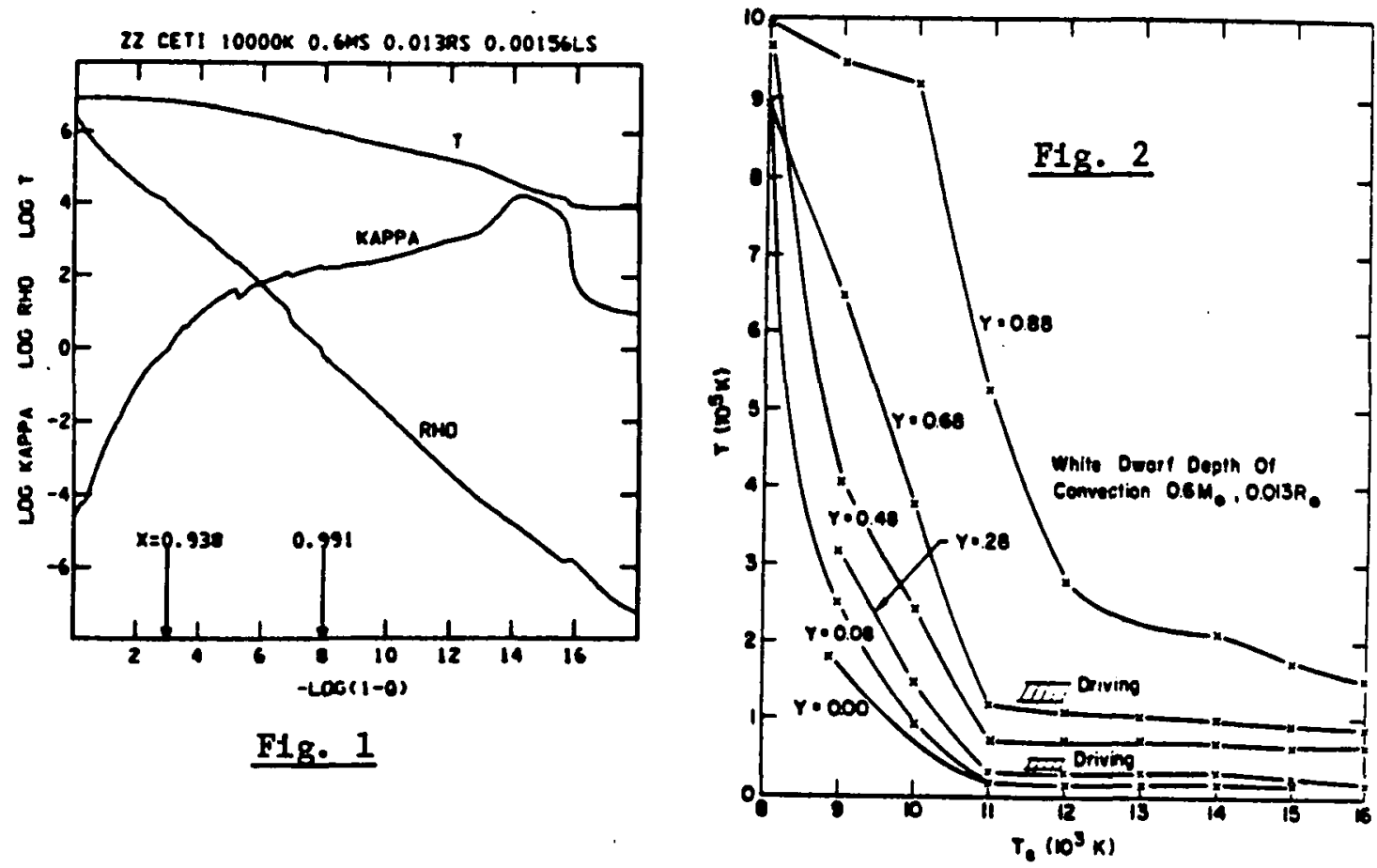

F1g. 1 - The temperature, opacity, and density as a function of surface mass fraction. Two values of $x=r / R$ are marked with arrows.

F1g. 2 - The depth in temperature to which the surface convection zone reaches as a function of effective temperature. Each " $x$ " marks a particular model and " $\mathrm{Y}$ " 1 s the mass fraction of helium.
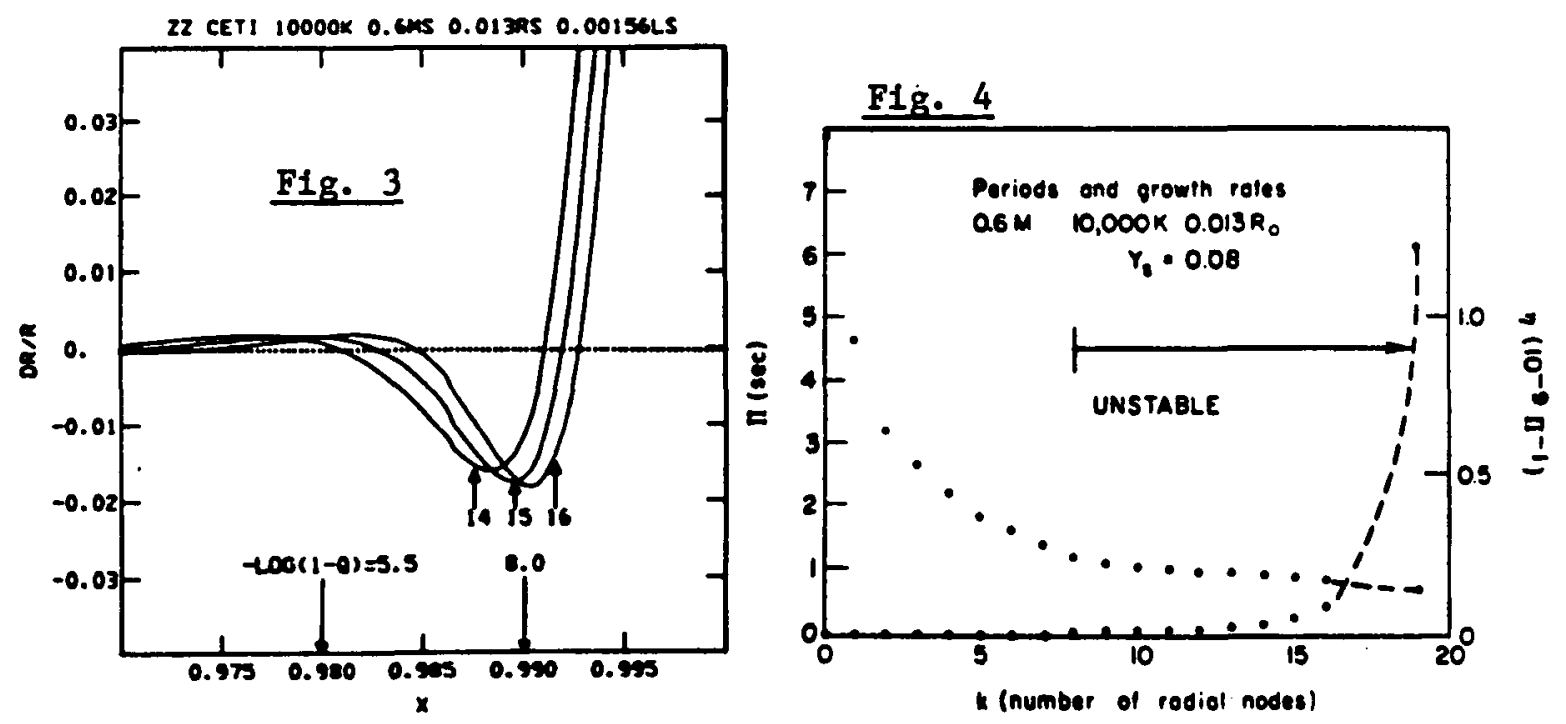

Fig. 3 - The Lagranglan elgenfunction as a function of $x=r / R$. Two values of the surface mass fraction are noted with arrows. "14", "15", and "16" is the number of nodes for that model.

F1g. 4 - The period in seconds, and growth rate ( $\triangle K E / K E$ per perlod) as a function of overtone. $k$ is the number of nodes. 
depending upon the composition. We find unstable modes in all envelopes lying in the strip and for all comositions $(Y=0.0$ to $Y=0.88$ ). The fact that the pure hydrogen $(Z=.02)$ models also pulsate is extremely important and we shall discuss its significance in the next section. As is well understood for the Cephelds (Cox, King, and Tabor 1973) the blue edge is a strong function of composition. For $Y=0.08$ it 11 es at $\sim 1.4 \times 10^{4} \mathrm{~K}$, while for $\Psi=.881 \mathrm{t}$ extends past $1.6 \times 10^{4} \mathrm{~K}$.

The radial eigenfunctions $(\delta r / r)$ for the 13th, 14th, and 15th overtones are shown in Figure 3 as a function of radius fraction, $x=r / R$. As one expects for such high order overtones, the motion is largest in the surface layers and damps out quickly as one goes deeper through numerous nodes. Figure 4 shows both the perlod (In seconds) and growth rates $(\triangle \mathrm{KE} / \mathrm{KE})$ for one model as a function of $k$. All of the low order overtones are stable and it is not until $k$ equals 7 that we find an instability. Nevertheless, the growth rates remain $s$ mall unt $11 \mathrm{k}$ has reached 15 and then grow rapidiy to a maximum of $1.25 \times 10^{-9}$ per $0.5 \mathrm{sec}$. This means that the pulsation will e-fold in kinetic energy every $4 \mathrm{x}$ $10^{8} \mathrm{sec}$ or $13 \mathrm{yr}$. In fact, the growth rate is still increasing at the last value of $k$, and $1 t$ could continue to grow for even higher overtones. Our limft to $k$ is set by the number of zones in the mesh (195) and we are currently redoing our analysis for some envelopes with 400 zones. This will allow us to study values of $k$ up to $\sim 40$. The integral of the work function ( $\triangle \mathrm{KE} / \mathrm{KE}$ per period) is given in Figure 5, and it shows where both the driving (positive values) and damping (negative values) occur in the envelope as a function of zone number. The jagged appearance of these curves is caused by interpolation in a real physics table; no analytic fit is used.

The instability in these envelopes is caused by the $K$ and $\gamma$ mechanisms. The driving in zones 145 to 155 and 170 to 180 1s caused by helium ionization at temperatures of $2 \times 10^{4} \mathrm{~K}$ to $5 \times 10^{4} \mathrm{~K}$ and $10^{5} \mathrm{~K}$ to $2 \times$ $10^{5} \mathrm{~K}$. An analogous graph for the pure hydrogen model would show driving at temperatures $\sim 4 \times 10^{4} \mathrm{~K}$. This particular envelope $\left(T=10^{4} \mathrm{~K}\right)$ was unstable. For larger effective temperatures, we find that the driving regions are located much closer to the surface at much smaller values of the surface mass fraction. At $T_{e} \sim 1.5 \times 10^{4} \mathrm{~K}$ there is no longer enough mass in the driving region to cause an instability.

\section{SUMMARY AND DISCUSSION}

The LNA radial pulsation analysis of DA dwarfs in the observed instability strip has shown that they are all unstable in their higher order overtones. However, the modes that we predict are unstable all have perlods that are 2 to 3 orders of magnitude shorter than those observed in the $\mathrm{ZZ}$ Ceti variables. This is perplexing since even the models calculated with a nearly pure hydrogen composition $(X=.98, Z=.02)$ have unstable modes so that even if all of the helium has had time to settle beyond the reach of a surface convection zone, the stars should st111 pulsate with short periods. Here we suggest a number of solutions to this dilemma.

First, it is still possible that a proper search for these periods has not yet been done. It 18 difficult to obtain and reduce data at the 


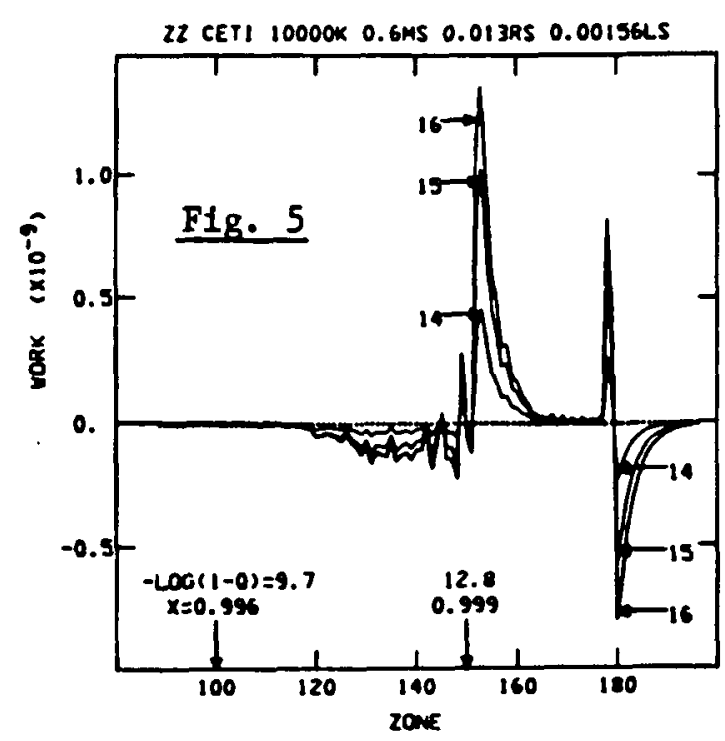

F18. 5 - The plot of the work function over one pulsation period as a function of zone number. Two values of the surface mass fraction and the radius fraction are denoted by arrows. Note that this region occurs much closer to the surface than that plotted in Figure 3.

$0.1 \mathrm{sec}$. rates necessary to discover these perlods and only Richer and Ulrich (1974) have reported on data taken w1th integration times shorter than 1 sec. Unfortunately, thelr power spectrum analysis gave incorrect results (McGraw 1979, private communication). Therefore, it could be posstble that these periods are there but have not been looked for with short enough integration times. Recently, a search was begun that will be able to detect periods as short as 0.2 sec. (McGraw and Starrfield 1979 , in preparation). At the present time, only the results for G117$B 15 A\left(T_{e} \approx 13,500\right)$ have been analyzed and they have proved to be negative. However, this variable is a known 22 Cet 1 star which is already pulsating at a period of $216 \mathrm{sec}$. (McGraw 1977) and it seems quite 11kely that once a variable star is trapped in one particular mode it cannot easily change and begin pulsating in another mode. Nonradial growth rates (Dziembowsk1 1979) may be larger than our radial growth rates. If this is true, then the stars in which to search for short period oscillations may be the current nonvariables in the instability strip. Another possible explanation for this discrepancy is that this star has zero helium content to below $150,000 \mathrm{~K}$ and is bluer than the pure hydrogen case blue edge.

We would like to thank Drs. J. McGraw, J. Llebert, H. Van Horn, R. Nather, E. Robinson, W. Dzlembowski, and D. Keeley for useful discussions on this work. S. Starrfield is grateful to P. Strittmatter for the hospitality of Steward Observatory and to P. Carruthers and A. N. Cox for the hospitality of the Los Alamos Scientific Laboratory and a generous allotment of computer t1me.

\section{REFERENCES}

Castor, J. I. 1971, Ap. J., 166, 109.

Cox, J. P., and Giuli, R. T. 1968, Princlples of Stellar Structure

(Gordon and Breach: New York).

Cox, A. N., King, D. S., and Tabor, J. E. 19.73, Ap. J., 184, 201.

Cox, A. N., Hodson, S. W., and Starrfield, S. 1979, in proceedings of the Stellar Nonradial and Nonlinear Pulsation Workshop, eds. H. A. Hill and $W$. Dziembowski (Springer-Verlag) in press.

Cox, A. N., and Tabor, J. E. 1976, Ap. J. Supp1., 31, 271. 
Dziembowsk1, W. 1977, Acta Astronomica, 27, 1.

Dzlembowsk1, W. 1979, In these Proceedings.

Fontalne, G. and Van Horn, H.M. 1976, Ap.J. Supp1., 31, 467.

Hamada, T., and Salpeter, E.E. 1961, AP.J., 134, 683.

Huebner, W.F., Merts, A.L., Magee, N.H., and Argo, M.F. 1977, LA-6760-M, Los Alamos Scient1fic Laboratory Report.

Kutter, G.S., and Sparks, W.M. 1972, Ap.J., 175, 407.

McGraw, J.T. 1977, Ph.D. Dissertation, Univ。 of Texas。

McGraw, J.T. 1979, Ap.J., 229, 203.

Nather, E. 1978, Pub. Ast. Soc. Pac., 90, 477.

Richer, H.B., and Ulrych, T.J. 1974, AP.J., 192, 719.

Starrfield, S., Truran, J.Wa, and Sparks, W.M. 1978, Ap.J., 226, 186. Stellingwerf, R.E. 1975, Ap.J., 195, 441. 